\title{
Factores que limitan all docente en la integración inclusiva de los niños y niñas con necesidades educativas especiales
}

\section{Factors that limit the teacher in the inclusive integration of children with special educational needs}

\author{
Lindo, Ivett Francisca; Zúniga-González, Carlos Alberto; Editor \\ Academico Dr. Angel Sol Sanchez
}

Ivett Francisca Lindo

ivett.lindo@post.unanleon.edu.ni

Ministerio de Educación (MINED)-Somotillo,

Nicaragua

(iD) Carlos Alberto Zúniga-González

carlos.zuniga@ev.unanleon.edu.ni

Universidad Nacional Autónoma de Nicaragua, León.,

Nicaragua

Editor Academico Dr. Angel Sol Sanchez

Colegio de posgraduados, México

Revista Iberoamericana de Bioeconomía y Cambio Climático

Universidad Nacional Autónoma de Nicaragua, León, Nicaragua ISSN-e: 2410-7980

Periodicidad: Semestral

vol. 2, núm. 1, 2016

czuniga@ct.unanleon.edu.ni

Recepción: 08 Marzo 2015

Aprobación: 25 Abril 2016

URL: http://portal.amelica.org/ameli/journal/394/3941750025/

DOI: https://doi.org/10.5377/ribcc.v2i1.5704

Autor de correspondencia: ivett.lindo@post.unanleon.edu.ni
Resumen: El presente ensayo se centró en revisar la implementación de estrategias que le faciliten al docente la integración de los niños y niñas con necesidades educativas asociadas o no a una discapacidad y es por eso que el Gobierno de Reconciliación DE Unidad Nacional (GRUM) en coordinación con el Ministerio de Educación (MINED) Nicaragua en consecuencia con los compromiso adquiridos dentro del marco de acción realizar acciones dirigidas a brindar una educación que satisfaga las necesidades educativas de todos y todas los niños, jóvenes y adultos. En conclusión la inclusión educativa presenta una complejidad que puede ser comprendida mejor si tiene al profesor como agente relevante y clave de este proceso. La actitud del docente con respectos a estas prácticas, en dependencia de su actitud puede constituirse en una barrera o en un agente facilitador. Por otra parte los recursos con que se cuenta facilitan o limita las posibilidades u oportunidades de generar espacios educativos más inclusivos.

Palabras clave: Educación, Inclusiva, Síndrome de Down, Discapacidad.

Abstract: This essay focused on reviewing the implementation of strategies that facilitate the teacher the integration of children with educational needs associated or not with a disability and that is why the Reconciliation Government of National Unity (GRUN) in coordination with the Ministry of Education (MINED) Nicaragua and consequently with the commitments acquired within the framework of action, carry out actions aimed at providing an education that meets the educational needs of all children, youth and adults. In conclusion, educational inclusion presents a complexity that can be better understood if it has the teacher as a relevant and key agent of this process. The teacher's attitude regarding these practices, depending on their attitude, can become a barrier or a facilitating agent. On the other hand, the resources available facilitate or limit the possibilities or opportunities to generate more inclusive educational spaces.

Keywords: Education, Inclusive, Down's syndrome, Disability; .

\section{NotAS DE AUTOR}




\section{INTRODUCCIÓN}

El modelo inclusivo es hoy en día, un referente para muchos contextos educativos que ven la necesidad de implementar estos principios al interior de sus comunidades educativas, dentro de este marco; existen muchos factores que están interviniendo en el éxito o fracaso de esta propuesta; un elemento que emerge con relevancia es la actitud del profesorado hacia la inclusión educativa, pues esta, puede facilitar la implementación o constituir una barrera para el aprendizaje y la participación del alumnado (Beltran, 1993; Both \& Ainscow, 2011; Cook \& Cameron, 2010).

Según autores Booth y Ainscow (2012) la inclusión educativa es un conjunto de procesos orientados a eliminar o minimizar las barreras que limitan el aprendizaje y la participación de todos los estudiantes asociados o no a una discapacidad. Sus dimensiones incorporan la cultura, política y práctica. Dentro de la cultura se plantea una comunidad escolar con valores y creencias compartidos y orientados a que todos aprendan implicando las escuelas en su conjunto, estudiantes, miembros del consejo escolar y familia.

Las políticas por su parte apuntan a focalizar a la inclusión como centro de desarrollo de las escuelas y constituyen un único marco que orienta los distintos apoyos para responder a la diversidad. A sí mismo, las prácticas aseguran que las actividades escolares favorecen la participación de todos y todas (De Boer et al., 2011; Eiseman et al., 2011; Home \& Timmons, 2009).

\section{ReVisión DE LA Literatura}

\section{Educación inclusiva en América Latina}

América Latina se caracteriza por tener sociedades muy desintegrada y fragmentada debido a la persistencia de la pobreza y a la gran desigualdad en la distribución de los ingresos, lo cual genera altos índices exclusión, el aumento de las desigualdades sociales, las segmentaciones espaciales y la fragmentación cultural de la población (Sola, 1997; Tilstone, 2003; Warnock 1978).

Durante la década de los 90 los países de América Latina iniciaron una serie de reforma educativa orientada a lograr el acceso universal a la educación básica y al mejoramiento de calidad y equidad sin embargo todavía persisten importantes desigualdades educativas (Jordan et al, 2010; Sainback, 1999; Sanhueza 2012).

La mayoría de los países adoptan en sus políticas y leyes los principios de la declaración de una educación para todos; pero en la práctica existen distintos factores que incluyen y discriminan a numerosos alumnos del sistema educativo. A pesar del gran avance alcanzado en el acceso a la educación aún no se ha alcanzado la universalización de la educación primaria y persisten problemas de equidad en la distribución y calidad de la oferta educativa y el acceso al conocimiento, los colectivos más excluidos son precisamente los que más necesitan la educación para superar su situación de desventaja o de vulnerabilidad; niños y niñas de zonas rurales aislados o de extrema pobreza, niños y niños con discapacidad (Alegre, 2000; Arnaiz, 2003; Stainback, 1999; Tilstone, 2003; Booth, 2012)

Actualmente no se ha logrado el pleno acceso a la educación básica; el mayor desafío es superar la desigualdad en la calidad de la oferta educativa y en los logros de aprendizaje. El aumento de la cobertura no ha sido acompañado de medios efectivos que garanticen la permanencia en el sistema educativo escolar en donde se vienen desarrollando estrategias y programas positivos para favorecer el acceso y la permanencia de los alumnos con necesidades educativas especiales asociadas o no a una discapacidad (Cook \& Cameron, 2010, De Boer et al., 2011).

Las desigualdades en función de origen socioeconómico son las más significativas en los países de América Latina y la pobreza está asociada a otros tipos de desigualdad como vivir en la zona rural o pertenecer a pueblos originarios, los niños y las niñas con necesidades educativas asociadas o no a una discapacidad son los 
que se encuentran excluidos debido a tantos factores como son las desigualdades económicas, discriminación atención integral, de la salud, entre otros y sobre todo en los centros escolares los docentes no cuenta con los medios tecnológicos suficientes para la atención de estos la desigualdad que sufren. Es preciso añadir el debilitamiento de las escuelas públicas en nuestro país Nicaragua y sobre todo en nuestro municipio Somotillo, Jordan (et al. (2009).

\section{Etapas fundamentales en el ejercicio del derecho}

Como consecuencia de una desigualdad en las escuelas públicas y privadas en términos de recursos docentes calificados y resultados de aprendizaje tienden a ampliarse especialmente en los más pobres aumentando la brecha social y reproduciendo la estratificación y fragmentación presentes en nuestras sociedades, Idol (2006).

Katarina Tomasevisky (UNESCO) señala que normalmente los países pasan por tres etapas fundamentales hacia el pleno ejercicio del derecho a la educación.

1. Conceder el derecho a la educación a todos aquellos que por diferentes causas personas con discapacidad, comunidades nómadas pero con opciones segregadas en escuelas especiales o programas diferenciados para esto que se incorporan a la educación.

2. Enfrentar el problema de la segregación educativa promoviendo la educación en las escuelas para todos.

3. Exigir la adaptación de la enseñanza a la diversidad de necesidades educativas del alumnado que son frutos de su descendencia social, cultural y de sus características individuales; en cuanto a motivaciones, capacidades e intereses.

En muchos países existen cierta confusión con el concepto de inclusión o educación inclusiva, como sinónimo de integración de niños y niñas con discapacidades u otros con necesidades educativas especiales a la escuela común. Es decir se está asimilando el movimiento de inclusión con el de integración cuando se trata de dos enfoques con una visión y foco distinto; porque esa confusión tiene como consecuencia que las políticas de inclusión se consideren como una responsabilidad de la educación especial, limitándose el análisis de la totalidad de exclusiones y discriminaciones que se dan al interior del sistema educativo e impidiendo el desarrollo de las políticas inclusivas integrales. La inclusión está relacionada con el acceso, la participación y logros de todos los alumnos con especial riesgo de ser excluidos o marginados por diferentes razones. Desde esa perspectiva, la inclusión es una política del MINED en su conjunto y no de las divisiones de educación especial en segundo lugar, el foco de atención es de naturaleza distinta (Jordan et al., 2009; Lindsay, 2010).

La preocupación de la integración ha estado más en transformar la educación especial, para apoyar los procesos de integración que cambiar la cultura y prácticas de las escuelas comunes para que sean capaces de atender a la diversidad del alumnado y eliminar los diferentes tipos de discriminación que tienen lugar al interior de ellos. Aunque muchas escuelas se ha producido los procesos de cambio como consecuencia de la incorporación de alumnos con necesidades educativas especiales, el movimiento de la integración no ha logrado alternar los sistemas educativos de forma significativa centrándose más en la atención individualizada de estos alumnos, programas individuales, estrategias, materiales diferenciados entre otros, que en modificar aquellos factores del contexto educativo y de la enseñanza que limita la participación y el aprendizaje no solo de los niños y jóvenes integrados sino de todo el alumnado. En el enfoque de la inclusión, por el contrario se considera que el problema no es el niño si no el sistema educativo y sus escuelas.

\section{Factores que facilitan o limitan el desarrollo de las prácticas educativas.}

I. Perspectiva profesional: los docentes en su práctica profesional no solo deben dominar los contenidos que imparten, sino que es necesario conocer a sus estudiantes, sus intereses, necesidades, deseos, sentimientos, lo 
cual se facilita el aprendizaje y la participación de todos los (as) estudiantes propiciando oportunidades de mayor inclusión educativa.

En la medida que el docente asuma esta doble responsabilidad, manifestara una actitud más positiva y humanista ante los estudiantes con distintas necesidades educativas. Ahora bien si la actitud del profesor hacia la inclusión educativa esta condicionadas con actitudes negativas pesimistas estas pueden obstaculizar sus prácticas inclusivas.

II. Experiencia profesional: el proceso pedagógico está condicionada por cinco aspectos.

a. La experiencia de los docentes.

b. Las características de los estudiantes.

c. El tiempo y recursos de apoyo.

d. La formación del docente.

e. El entorno o contexto social del estudiante.

En este sentido es posible precisar que la experiencia de los docentes influye en el desarrollo de la personalidad del estudiante, sus años de trabajo, las experiencias vinculadas a las prácticas inclusivas favorecen su labor como profesional en las aulas de clase, sin embargo en nuestro país Nicaragua se carece de docentes formados o capacitados en este campo.

Los estudios indican que los profesores responden de manera diferente según el tipo de discapacidad sin considerar el diagnostico entregado. Así los docentes se integran de manera más positiva a la intención a niños (as) con discapacidades intelectuales, síndrome de Down, discapacidades físicas, parálisis cerebral entre otros. A pesar de constar con el apoyo de nuestro gobierno de Reconciliación y Unidad Nacional (GRUN) se carece de recursos que facilitan el acceso educativo ya sean los recursos humanos, personas con capacidades intelectuales expertas en estas áreas para trabajar con cada uno de estos niños (as) debido a que la educación regular no tiene la formación necesaria para atender estas necesidades educativas con eficacia.

III. Las características de los estudiantes: para detallar este factor, es necesario saber que los estudiantes están condicionados por necesidades educativas permanentes o transitorias que afectan el proceso de Enseñanza - Aprendizaje. Es necesario definir lo que son las necesidades educativas especiales.

Son aquellas dificultades que se presentan durante toda la trayectoria escolar y vida en general en ella se encuentran las deficiencias visuales, auditivas, motoras e intelectuales y los problemas de aprendizaje los cuales merecen una atención pedagógica específica para cada estudiante.

IV. La discriminación: En la actualidad existe como un flagelo social, un alto índice de rechazo a las personas con necesidades educativas especiales desde la familia y la sociedad afectándoles el autoestima, los padres tienden a sobreproteger a sus hijos lo que impide el acceso a las aulas de clase.

V. Otro de los factores que influyen a la atención de los niños con necesidades educativas especiales son los: recursos y materiales, los cuales continúan siendo percibidos por el profesorado como una dificultad para el desarrollo de las prácticas educativas.

Se necesita por tanto realizar Adecuaciones Curriculares, entiéndase por ellas a las estrategias educativas generalmente dirigido a estudiantes con necesidades educativas asociados o no a discapacidad, para alcanzar los propósitos de la enseñanza. Este consiste en adaptar el currículo de un determinado nivel educativo para que sea más accesible a un estudiante de un grupo de estudiante en su proceso de escolarización.

Para realizar Adecuaciones Curriculares se necesita de un diagnostico educativo de las necesidades del estudiante, las capacidades y desarrollo general, los recursos escolares y extraescolares con que se cuenta y conocer el ámbito familiar. Si el estudiante recibe tratamiento educativo extraescolar con algún otro profesional las adecuaciones curriculares y el programa de intervención educativa deben coordinarse para fortalecer su aprendizaje y evitar que las exigencias en las tareas escolares no sobrepasen la capacidad en los niños (as) con necesidades educativas especiales (Sánchez et al, 2008).

Es necesario respetar el ritmo de aprendizaje del alumno y aprovechar su interés facilitando el aprendizaje.

Estrategias metodológicas del gobierno de reconciliación y unidad nacional (GRUN) 
Desde la década de los noventa el Gobierno de reconciliación y unidad nacional ha venido desarrollando un sin número de estrategias y programas que favorezcan el acceso y permanencia de los niños y niñas con necesidades educativas asociadas o no a una discapacidad. Entre ellas tenemos: Las cinco políticas Educativas y Metodologías Pedagógicas para la atención de los niños(as) con necesidades educativas especiales a los diferentes docentes del país y la nueva transformación curricular flexible que favorece el aprendizaje del niño (a) (Eisenman et al., 2011; Horne \& Timmons 2009; Huang \& Diamond, 2009)).

En consecuencias con los compromisos adquiridos dentro del marco de acción de Dakar (2000) ha venido realizando acciones dirigidas a brindar una educación que satisfaga las necesidades educativas de los niños (Lindsay, 2010).

\section{ConCLUSIÓN}

En conclusión todos estos factores influyen en la atención de los niños con necesidades educativas las cuales continúan siendo percibidas por el profesorado como una dificultad para el desarrollo de las prácticas educativas.

La perspectiva profesional no está sustentando la necesidades educativas en los niños(a).la falta de atención individualizada a cada niño según su necesidad educativa y la discriminación, la falta de recursos didácticos y del medio.

Con todo lo antes expuesto se puede afirmar que la generación de un proceso más inclusivo requieren de tiempo y compromiso necesario para integrar y asumir cambios que demanden la implementación de un sistema abierto a la diversidad; esto implica llevar a cabo las transformaciones curriculares y humanas que se originan desde la creación de políticas inclusivas, desde una cultura que acoge con prácticas pedagógicas que responden a las distintas formas de aprendizaje del estudiante.

\section{REFERENCIAS}

Alegre, O. M. (2000). Diversidad Humana y Educación. Málaga: Aljibe, 23-50.

Beltrán Llera, J. (1993). Procesos, estrategias y técnicas de aprendizaje. Editorial Sintesis, SA Madrid.

Booth, T., \& Ainscow, M. (2012). Guía para la inclusión educativa: desarrollando el aprendizaje y la participación en las escuelas. Santiago, Chile: CSIE-FCF.

Cook, B. G., \& Cameron, D. L. (2010). Inclusive teachers' concern and rejection toward their students: Investigating the validity of ratings and comparing student groups. Remedial and Special Education, 31(2), 67-76.

De Boer, A., Pijl, S. J., \& Minnaert, A. (2011). Regular primary schoolteachers' attitudes towards inclusive education: A review of the literature. International journal of inclusive education, 15(3), 331-353.

Eisenman, L. T., Pleet, A. M., Wandry, D., \& McGinley, V. (2011). Voices of special education teachers in an inclusive high school: Redefining responsibilities. Remedial and special education, 32(2), 91-104.

Horne, P. E., \& Timmons, V. (2009). Making it work: Teachers' perspectives on inclusion. International journal of inclusive education, 13(3), 273-286.

Huang, H. H., \& Diamond, K. E. (2009). Early childhood teachers' ideas about including children with disabilities in programmes designed for typically developing children. International Journal of Disability, Development and Education, 56(2), 169-182.

Idol, L. (2006). Toward inclusion of special education students in general education: A program evaluation of eight schools. Remedial and Special education, 27(2), 77-94.

Jordan, A., Glenn, C., \& McGhie-Richmond, D. (2010). The Supporting Effective Teaching (SET) project: The relationship of inclusive teaching practices to teachers' beliefs about disability and ability, and about their roles as teachers. Teaching and teacher education, 26(2), 259-266. 
Ivett Francisca Lindo, et al. Factores que limitan al docente en la integración inclusiva de los N...

Jordan, A., Schwartz, E., \& McGhie-Richmond, D. (2009). Preparing teachers for inclusive classrooms. Teaching and teacher education, 25(4), 535-542.

Lindsay, G. (2010). Intervención en el lenguaje en una escuela inclusiva. In Dificultades del lenguaje, colaboración e inclusión educativa: manual para logopedas, psicopedagogos y profesores (pp. 93-104).

Sánchez Bravo, A., Díaz Flores, C., Sanhueza Henríquez, S., \& Friz Carrillo, M. (2008). Percepciones y actitudes de los estudiantes de pedagogía hacia la inclusión educativa. Estudios pedagógicos (Valdivia), 34(2), 169-178.

Sanhueza, S., Granada, M. y Bravo, L (2012) Actitudes del profesorado de Chile y Costa Rica hacia la inclusión educativa. Revista Cadernos de Pesquisa.

STAINBACK, S., \& STAINBACK, W. (1999). Aulas inclusivas, Madrid, Narcea. VLACHOLL, AD.

Sola, T. (1997). La formación inicial y su incidencia en la educación especial. En Sánchez Palomino, A. Y J. Torres González, Educación especial I. Una perspectiva curricular, organizativa y profesional. Madrid: Pirámide.

Tilstone, CH., Lani, F. Y Richard, R. (2003). Promoción y desarrollo de prácticas educativas inclusivas. Madrid: Eos

Warnock Report (1978). Report of the committee of enquiry into the education of handicapped children and young people. London: Her Majesty's Stationary Office.

Arnaiz, P. (2003). Educación inclusiva: una escuela para todos. Málaga: aljibe, 77. 